\title{
The protein folding network
}

\author{
Francesco Rao and Amedeo Caflisch $^{*}$ \\ Department of Biochemistry, University of Zurich, \\ Winterthurerstrasse 190, CH-8057 Zurich, Switzerlandi \\ tel: +411635 55 21, fax: +41163568 62, e-mail: caflisch@bioc.unizh.ch
}

\begin{abstract}
The conformation space of a 20 -residue antiparallel $\beta$-sheet peptide, sampled by molecular dynamics simulations, is mapped to a network. Conformations are nodes of the network, and the transitions between them are links. The conformation space network describes the significant free energy minima and their dynamic connectivity without projections into arbitrarily chosen reaction coordinates. As previously found for the Internet and the World-Wide Web as well as for social and biological networks, the conformation space network is scale-free and contains highly connected hubs like the native state which is the most populated free energy basin. Furthermore, the native basin exhibits a hierarchical organization which is not found for a random heteropolymer lacking a predominant free-energy minimum. The network topology is used to identify conformations in the folding transition state ensemble, and provides a basis for understanding the heterogeneity of the transition state and denaturated state ensemble as well as the existence of multiple pathways.
\end{abstract}

Keywords: complex networks, protein folding, energy landscape, transition state, denaturated state ensemble, multiple pathways

Proteins are complex macromolecules with many degrees of freedom. To fulfill their function they have to fold to a unique three-dimensional structure (native state). Protein folding is a complex process governed by noncovalent interactions involving the entire molecule. Spontaneous folding in a time range of microseconds to seconds 1 can be reconciled with the large amount of conformers by using energy landscape analysis 2.3 .4 . The main difficulty of this analysis is that the free-energy has to be projected on arbitrarily chosen reaction coordinates (or order parameters). In many cases a simplified representation of the free-energy landscape is obtained where important informations on the non-native conformation ensemble and the folding transition state ensemble are hidden. Moreover, the possible transitions between free-energy minima cannot be displayed in such projections which hinder the study of pathways and folding intermediates. The characterization of the free-energy minima and the connectivity among them, i.e., possible transitions between minima, for peptides and proteins is still an unresolved problem.

In the last five years many complex systems, like the World-Wide Web, metabolic pathways, and protein structures have been modeled as networks ${ }^{5.6 .7}$. Intriguingly, common topological properties have emerged from their organization ${ }^{8}$. A description of the potential energy landscape without the use of any projection has been given in terms of networks for a Lennard-Jones cluster of atoms 9 .

Here, we introduce complex network analysis 8 to study the conformation space and folding of beta3s, a designed 20-residue sequence whose solution conformation has been investigated by NMR spectroscopy $\stackrel{10}{ }$. The NMR data indicate that beta3s in aqueous solution forms a monomeric (up to more than $1 \mathrm{mM}$ concentration) triplestranded antiparallel $\beta$-sheet (Fig. 1, bottom), in equilibrium with the denaturated state 10 . We have previously shown that in implicit solvent ${ }^{11}$ molecular dynamics sim- ulations beta3s folds reversibly to the NMR solution conformation, irrespective of the starting conformation ${ }^{12.13}$. We consider conformations sampled by molecular dynamics simulations and the transitions between them as the network nodes and links, respectively. The network analysis allows to identify the topological properties that are common to both beta3s, which folds to a unique threedimensional structure $\stackrel{10.14}{ }$, and a random heteropolymer which lacks a single preferential conformation like the native state despite it has the same residue composition as beta3s. These properties include the presence of several free-energy minima and highly connected conformations (hubs). On the other hand, a hierarchical modularity 15 in the proximity of the native state is peculiar of a folding sequence.

\section{MODEL AND METHODS}

Molecular dynamics simulations The simulations and part of the analysis of the trajectories were performed with the program CHARMM 17 . Beta3s was modeled by explicitly considering all heavy atoms and the hydrogen atoms bound to nitrogen or oxygen atoms (PARAM19 force field ${ }^{17}$ ). A mean field approximation based on the solvent accessible surface was used to describe the main effects of the aqueous solvent on the solute $\frac{11}{}$. The two parameters of the solvation model were optimized without using beta3s. The same force field and implicit solvent model have been used recently in molecular dynamics simulations of the early steps of ordered aggregation 18 , and folding of structured peptides ( $\alpha$-helices and $\beta$-sheets) ranging in size from 15 to 31 residues 11.12 .19 , as well as small proteins of about 60 residues ${ }^{20,21}$. Despite the absence of collisions with water molecules, in the simulations with implicit solvent the separation of time scales is comparable with that observed experimentally. Helices fold in about $1 \mathrm{~ns}^{22}, \beta$ - 


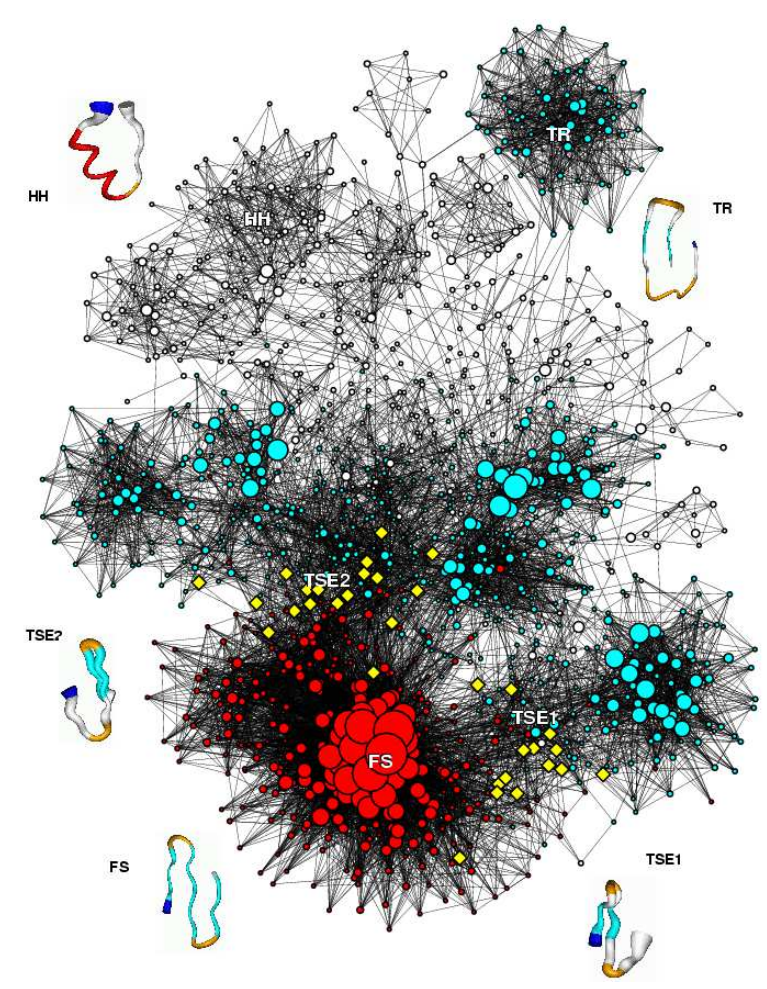

FIG. 1: Beta3s conformation space network. The size and color coding of the nodes reflect the statistical weight $w$ and average neighbor connectivity $k_{n n}$, respectively. White, cyan, and red nodes have $k_{n n}<30,30 \leq k_{n n} \leq 70$, and $k_{n n}>70$, respectively. Representative conformations are shown by a pipe colored according to secondary structure: white stands for coil, red for $\alpha$-helix, orange for bend, cyan for strand and the N-terminus is in blue. The variable radius of the pipe reflects structural variability within snapshots in a conformation. The yellow diamonds are folding TS conformations (TSE1, TSE2, see text for details) characterized by a connectivity/weight ratio $k / 2 \tilde{w}>0.3$, a clustering coefficient $C<0.3$, and $60<k_{n n}<80$. This figure was made using visone (www.visone.de) and $M O L M O L^{16}$ visualization tools.

hairpins in about $10 \mathrm{~ns}^{22}$ and triple-stranded $\beta$-sheets in about $100 \mathrm{~ns}^{13}$, while the experimental values are $\sim 0.1 \mu \mathrm{s}^{23}, \sim 1 \mu \mathrm{s}^{23}$ and $\sim 10 \mu \mathrm{s}^{10}$, respectively. Recently, four molecular dynamics simulations of beta3s were performed at $330 \mathrm{~K}$ for a total simulation time of $12.6 \mu \mathrm{s}^{14}$. There are 72 folding events and 73 unfolding events and the average time required to go from the denatured state to the folded conformation is $83 \mathrm{~ns}$. The $12.6 \mu \mathrm{s}$ of simulation length is about two orders of magnitude longer than the average folding or unfolding time, which are similar because at $330 \mathrm{~K}$ the native and denatured states are almost equally populated 14 . For the network analysis the first $0.65 \mu$ s of each of the four simulations were neglected so that along the $10 \mu \mathrm{s}$ of simulations there are a total of $5 \times 10^{5}$ snapshots because coordinates were saved every $20 \mathrm{ps}$. The sequence of the random heteropolymer is a randomly scrambled version of the beta3s sequence with the same residue composition. It was simulated for $2 \mu \mathrm{s}$ and $10^{5}$ snapshots were saved. The conditions for the molecular dynamics simulations, i.e., force field, solvation model, temperature, and time interval between saved snapshots were the same for both peptides.

Construction of the protein folding network To define the nodes and links of the network the secondary structure was calculated ${ }^{24}$ for each snapshot (Cartesian coordinates of the atomic nuclei) saved along the molecular dynamics trajectory. A "conformation" is a single string of secondary structure ${ }^{24}$, e.g., the most populated conformation for beta3s (FS in Fig. 1) is -EEEESSEEEEEESSEEEE- where "E", "S", and "-" stand for extended, turn, and unstructured, respectively. There are 8 possible "letters" in the secondary structure "alphabet". Since the N- and C-terminal residues are always assigned an "-" 24 a 20 -residue peptide can assume $8^{18} \simeq 10^{16}$ conformations. Conformations are nodes of the network and the transitions between them are links. A weight $\tilde{w}$ is assigned to each node to take into account the free-energy of each conformation and is equal to the number of snapshots with a given secondary structure string. The statistical weight $w$ of a node is equal to the weight normalized by the total number of snapshots in the simulations $\left(5 \times 10^{5}\right.$ and $10^{5}$ for beta3s and the random heteropolymer, respectively). Considering all the conformations visited during a $\mu s$ - scale simulation can yield to a computationally intractable network size. For this reason we used for the network analysis the 1287 conformations of beta3s with significant weight ( $\tilde{w} \geq 20$ per conformation). Two nodes are connected by an undirected link (and called neighbors) if they either include a pair of snapshots that are visited within 20 ps or they are separated by one or more conformations with less than 20 snapshots each. For the $2 \mu$ s of the random heteropolymer a threshold of $\tilde{w} \geq 4$ was used, so that $w \geq 4 \times 10^{-5}$ as in the beta3s network. The choice of a threshold value is somewhat arbitrary but the network properties are robust for a large range of threshold values (see Supplementary material).

The properties of the network are robust also with respect to the length of the simulation time and the definition of the nodes. The topological properties are independent from simulation lengths if one considers more than $2 \mu s$. The correlation between statistical weight and connectivity, as well as power-law behavior of the connectivity distribution and $1 / k$ behavior of the clustering coefficient distribution (see below) are essentially identical after 2,4 , and $10 \mu s$. As an example, the exponent of the power-law is 2.0 for the beta3s networks based on 2, 4 and $10 \mu s$ of simulation time. Defining nodes by grouping snapshots according to root mean square deviations (RMSD) in coordinates of $\mathrm{C}_{\alpha}-\mathrm{C}_{\beta}$ atoms yields the same overall properties i.e., power-law distribution of the links (with similar $\gamma$ value) and $1 / k$ tail of the clustering distribution. Grouping snapshots according to secondary structure motifs does not require the use of an arbitrarily 
chosen RMSD cutoff, and is able to capture the fluctuations of partially structured conformations ${ }^{24}$.

Evaluation of $\mathbf{P}_{\text {fold }}$ The TS ensemble can be defined as the set of structures which have the same probability of folding $\left(P_{\text {fold }}\right)$ or unfolding in trajectories started with varying initial conditions 25 . For each putative TS conformation, the probability to fold before unfolding was calculated by 100 very short trajectories at $330 \mathrm{~K}$ started from ten snapshots within a node. The only difference between the ten runs was the seed for the random number generator used for the initial assignment of the atomic velocities. A trajectory was considered to lead to folding (unfolding) if it visits first structures with a fraction of native contacts $Q>22 / 26(Q<4 / 26)^{12}$.

\section{RESULTS AND DISCUSSION}

To study the conformation space network of polypeptides we concentrate on the analysis of topology, i.e., on the study of the connectivity between different conformations, leaving for a later study the analysis of transition rates. We have investigated the network topologies of several peptides but on this paper we focus on beta3s and the random scrambled version of it. Additional details can be find in the Supplementary material where the network properties of another structured peptide and a glycine homopolymer are presented.

Conformation space network of a structured peptide The conformation space network and relevant structures of beta3s are shown in Fig. 1. The group of nodes at the bottom of Fig. 1 (red nodes) represents the native state basin (FS). The native basin is connected to a wide region of nodes with significant native content (cyan circles in the middle of Fig. 1). Although many heterogeneous routes can be taken to reach the folded state (in agreement with lattice simulations ${ }^{26.27}$ ), most of the folding events have common structural features that define two average folding pathways. The less frequented average pathway (see Ref13 but also the density of transitions in Fig 1 bottom right) consists of conformations that have the N-terminal hairpin formed while the Cterminal strand is mostly unstructured with non-native hydrogen bonds at the turn (TSE1 in Fig. 1). The second and most frequented average pathway includes conformations with a well formed C-terminal hairpin while the N-terminal strand is disordered (TSE2 in Fig. 1), namely it can be out-of-register or mostly unstructured. It is interesting to note that the same two folding pathways were observed experimentally for a 24-residue peptide with the same folded state as beta $3 \mathrm{~s}^{28}$. Furthermore multiple folding pathways have recently been detected by kinetic analysis of a $\beta$-sandwich protein ${ }^{29}$.

The denatured state ensemble is very heterogeneous and includes high enthalpy, high entropy conformations (e.g., the partially helical conformations, denoted $\mathrm{HH}$ in
Fig. 1) but also low enthalpy, low entropy conformations (e.g., the curl-like trap, TR). The former are loosely linked clusters of conformations with similar secondary structure (see Tab. 1) which are characterized by an unfavorable effective energy (sum of peptide potential energy and solvation energy) and fluctuating unstructured residues (e.g., the terminal of the helix shown on top left of Fig. 1). On the contrary, low enthalpy, low entropy traps form tightly linked clusters with almost identical secondary and tertiary structure, favorable effective energy (similar to the one of the native structure, see Tab. 1) and no fluctuating residues (e.g., Fig. 1, top right). Taken together, these results indicate that FS is entropically favored over low enthalpy conformations like TR, i.e., FS has more flexibility than TR. A possible explanation is that the C-terminal carboxy is involved in four hydrogen bonds in TR (with the backbone NH's of residues 4-7), whereas both termini undergo rather large fluctuations in FS. In addition, a more favorable van der Waals energy in TR is consistent with a denser packing in TR than in FS.

TABLE 1: Energetic comparison of folded and denaturated state. The free-energy of conformation $i$ is $\mathcal{F}_{i}=-k_{B} T \log \left(w_{i}\right)$, where $w_{i}$ is the probability along the trajectory to find the peptide in the conformation $i$.

\begin{tabular}{|c|c|c|}
\hline Folded state (FS) & $\langle\mathcal{E}\rangle^{a}$ & $\Delta \mathcal{F}^{b}$ \\
\hline -EEEESSEEEEEESSEEEE- & -7.6 & 0 \\
\hline -EEE-STTEEEEESSEEEE- & -8.6 & 0.1 \\
\hline -EEEESSEEEEE-STTEEE- & -8.4 & 0.5 \\
\hline -EEE-STTEEEE-STTEEE- & -9.2 & 0.7 \\
\hline \multicolumn{3}{|l|}{ Helical conformations $(\mathrm{HH})$} \\
\hline ---нннннннннне------- & 0.9 & 3.1 \\
\hline -нннннннннннннь------- & -1.9 & 3.3 \\
\hline ---нннннннннннтТ----- & 0.7 & 3.5 \\
\hline ---ннннннннннн------- & 0.5 & 3.7 \\
\hline -нннннннннннннтТ----- & -0.8 & 3.7 \\
\hline --ТТ--Ннннннннннннн- & -0.8 & 3.8 \\
\hline \multicolumn{3}{|l|}{ Curl-like trap (TR) } \\
\hline ---SSGGG-EEE-STTTEE- & -7.8 & 3.4 \\
\hline ---SSSS--EEE-STTTEE- & -7.0 & 3.5 \\
\hline ---S-GGG-EEE-STTTEE- & -9.3 & 3.7 \\
\hline ---SSGGG-EEE-SGGGEE- & -9.6 & 3.7 \\
\hline ---SSTTT-EEE-STTTEE- & -8.4 & 3.7 \\
\hline \multicolumn{3}{|c|}{$\begin{array}{l}{ }^{a} \text { Average effective energy } \\
{ }^{b} \text { Free-energy relative to the most populated conformation. All } \\
\text { values are in kcal } / \text { mol. The conformational entropy of the peptide } \\
\text { is equal to }(\langle\mathcal{E}\rangle-\mathcal{F}) / T \text {. Note that the curl-like traps are entropi- } \\
\text { cally penalized with respect to the native state. }\end{array}$} \\
\hline
\end{tabular}

Note that the network description of non-native conformations is more detailed than the one obtained by projecting the free energy surface on progress variables (e.g., based on fraction of native contacts). In such projections, for low values of the fraction of native contacts structures as diverse as helices and the curl-like conformations mentioned above are not distinguished. Even 
the ensemble with half of the native contacts is heterogeneous and hard to classify. Using as reaction coordinate the RMSD (with respect to a given structure) or the radius of gyration is even less selective. Only when a clever combination of variables is used it is possible to have a more detailed description of the free-energy landscape. The network description of the conformation space gives a synthetic and systematic view of all the possible conformations accessed by the system and their transitions. By considering the statistical weight of the nodes a thermodynamical description of the system is obtained.

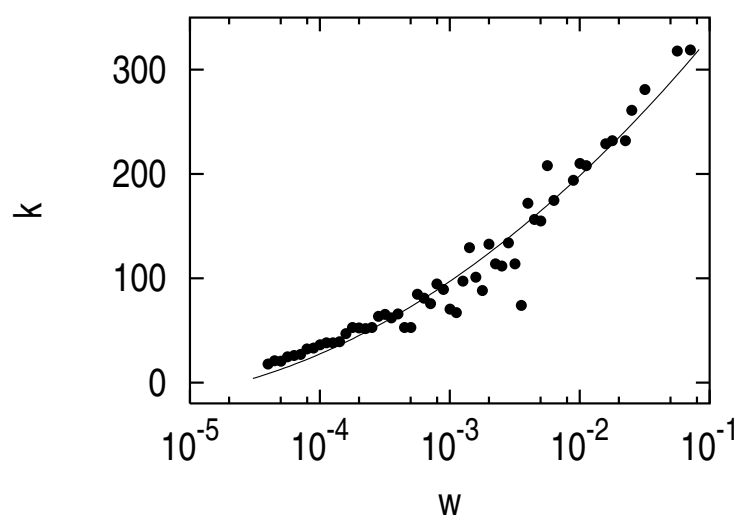

FIG. 2: Correlation between the statistical weight $w$ and the connectivity $k$ for beta3s. The connectivity is proportional to $\log ^{2}(w)$ with a correlation coefficient of 0.88 (solid line). The correlation and the fit are calculated over all nodes of the network but in the figure logarithmic binning is applied to reduce noise.

The high correlation between the statistical weight of a node and its number of links (Fig. 2) shows that the most connected nodes are also low lying minima on the freeenergy landscape. This indicates that the conformation space network describes the significant free energy minima and their dynamic connectivity, without projection, where highly populated nodes are minima of free-energy and the set of nodes densely connected to them make up the basins of such minima.

Folding and network topology The average neighbor connectivity $k_{n n}$ of beta3s (Fig. 3a), i.e., the average number of links of the neighbors of a given node, is rather heterogeneous, highlighting the presence of different connection rules in different regions of the network. This is not the case for the random heteropolymer (Fig. 3b) whose basins have organization and statistical weight similar among each others as previously found for most homopolymers 30 . Note that for beta3s the native state is well discriminated by $k_{n n}$ (red nodes in Fig. 1 and top band in Fig. 3a).

The connectivity distribution of conformation space networks shows a well pronounced power-law tail $P(k)=$ $k^{-\gamma}$ with $\gamma=2.0$ for both beta3s and the random heteropolymer (Fig. 4a) as well as another structured peptide ${ }^{31}$ and homoglycine, i.e. (Gly) 20 (see Supplementary material). The power-law is due to the presence of few largely connected "hubs" while the majority of the nodes have a relatively small number of links 32 . This behavior has been previously observed for several biological ${ }^{5}$, social $^{33}$ and technological networks ${ }^{6}$, which in the literature take the name of scale-free networks. In terms of free-energy this means that only a few low lying minima are present but they act as "hubs" with a large number of routes to access them.

The average clustering coefficient $C$ is a measure of the probability that any two neighbors of a node are connected. Beta3s and the heteropolymer have $C$ values of 0.49 and 0.28 , respectively. These values are one order of magnitude larger than random realizations of the two networks with the same amount of nodes and links. The native basin of beta3s includes the nodes with the largest number of links of the network. These nodes give rise to the $1 / k$ tail of the clustering distribution (Fig. $4 b)$, i.e., an inherently hierarchical organization ${ }^{15}$ of the conformations in the native basin of beta3s. Such organization is not observed for the non-native region of beta3s and the random heteropolymer. Note that the powerlaw scaling of the connectivity distribution can be considered as a general property of free-energy landscapes of polypeptides, whereas a hierarchical organization of the nodes reflects a single pronounced free-energy basin of attraction (like the native state).
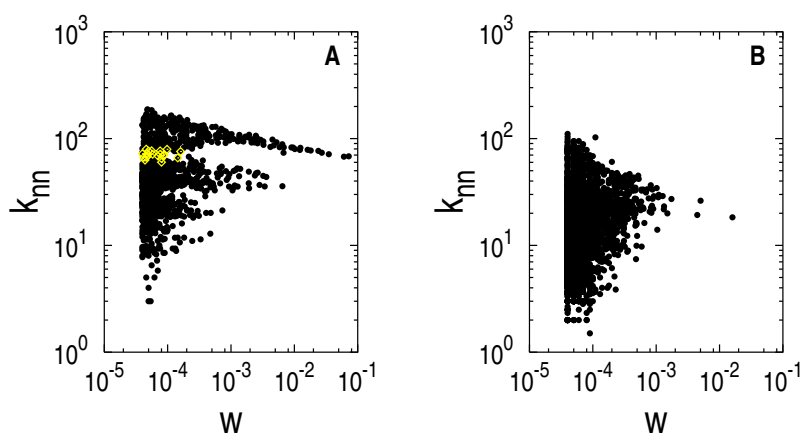

FIG. 3: Average neighbor connectivity $k_{n n}$ plotted as a function of the statistical weight for the 1287 nodes of beta3s (A) and for the 2658 nodes of the random heteropolymer (B). $k_{n n}$ of node $i$ is the average number of links of the neighbors of node $i$. The yellow diamonds are folding transition state conformations (see also Fig. 1 and text) characterized by a connectivity/weight ratio $k / 2 \tilde{w}>0.3$, a clustering coefficient $C<0.3$, and $60<k_{n n}<80$.

Transition state ensemble As mentioned above folding is a complex process with many degrees of freedom involved and it is difficult (or even not possible) to define a single reaction coordinate to monitor folding events 34,35 . Hence, it is very difficult to isolate transition state (TS) conformations from equilibrium sampling. The TS conformations are saddle points, i.e., local maxima with respect to the reaction coordinate for folding and local min- 

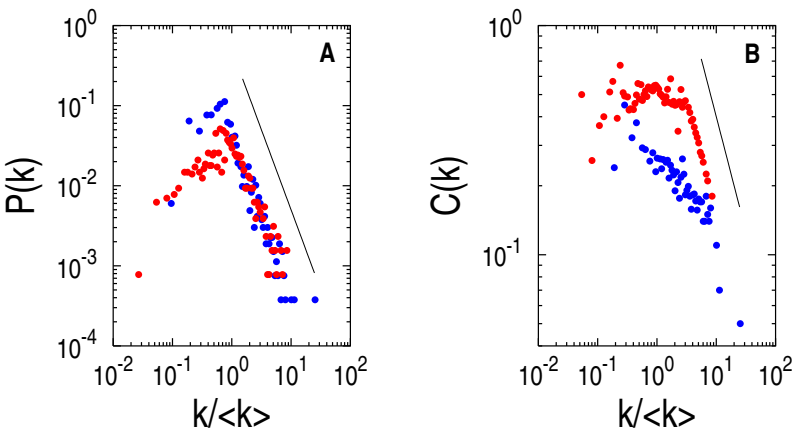

FIG. 4: Topological properties of conformation space networks. Red and blue data points are plotted for beta3s and a random heteropolymer, respectively. For a direct comparison, the connectivity $k$ is normalized by the average connectivity $\langle k\rangle$ of each network. Logarithmic binning is applied to reduce noise. (A) The connectivity distribution $P(k)$ is the probability that a node (conformation) has $k$ links (neighbor conformations). The straight line corresponds to a power-law fit $y=x^{-\gamma}$ on the tail of the distribution with $\gamma=2.0$. (B) The clustering coefficient $C$ describes the cliques of a node. For node $i$ it is defined as $C_{i}=\frac{2 n_{i}}{k_{i}\left(k_{i}-1\right)}$, where $k_{i}$ is the number of neighbors of node $i$ and $n_{i}$ is the total number of connections between them. Values of $C$ are averaged over the nodes with $k$ links. The straight line corresponds to a power-law fit $y=x^{-1}$ on the tail of the distribution of beta3s.

ima with respect to all other coordinates. For this reason we identified the nodes with a high connectivity/weight ratio $k_{i} / 2 \tilde{w}_{i}$ and low clustering coefficient value $C_{i}$ as putative TS conformations. The former criterion guarantees that these nodes are accessed and exited, most of the time, by a different route, i.e., they can be directly reached from different conformations of the network space. The low clustering coefficient value guarantees that the neighbors of these conformations are likely to be disconnected. These two conditions are necessary but not sufficient because they do not distinguish folding TS conformations from saddle points between unfolded conformations. Since the folding TS conformations are linked to both nodes in the native state (having large number of links) and in the denatured state (small/intermediate number of links), we speculated that folding TS conformations should have values of the average neighbor connectivity $k_{n n}$ within a certain range. For nodes with high connectivity/weight ratio and low clustering coefficient, a remarkable correlation of 0.89 was found between the average neighbor connectivity $k_{n n}$ and $P_{\text {fold }}$ (Fig. 5), which is the probability of a given conformation to fold before unfolding 25 . A $P_{\text {fold }}$ value close to 0.5 is expected for conformations on top of the folding TS barrier ${ }^{21}$ and the correlation suggests that network properties can be used to predict folding TS conformations. These are shown in Fig. 1 and 3a with yellow diamonds. As discussed above two main average folding pathways are observed. The less frequented one is characterized by a transition state ensemble of conformations with the first hairpin in a native form (residues 1-13) and a bend in correspondence of the the second native turn (residues 14-15). The C-terminal residues form a straight structure with almost no contacts, either native or non-native. The second average pathway shows a transition state with the second native harpin formed (residues 7-20) and a bend in correspondence of the the first native turn (residues 5-6). Such a symmetrical behavior is presumably due to the simplicity and symmetry of the native conformation as well as the symmetry in the sequence (sequence identity of $67 \%$ between the two hairpins). The folding TS conformations of beta3s form an heterogeneous ensemble with $\mathrm{C}_{\alpha}$ root mean square deviations within contributing structures between 3 and $6 \AA$ A. In contrast to previous molecular dynamics studies in which progress variables based on fraction of native contacts were used to describe TS conformations ${ }^{12.36}$, the network properties yield a description of the folding TS ensemble (Fig. 1) which does not depend on the choice of reaction coordinates. Interestingly, the folding TS conformations of beta3s have about one-half of the native contacts formed but this is not a sufficient criterion (Table S1 in Supplementary material). Moreover, there is no correlation between the fraction of native contacts and the probability of folding. As a control, $P_{\text {fold }}$ values smaller than 0.15 were obtained for five nodes with an average fraction of native contacts similar to the folding TS conformations but low connectivity/weight ratio and/or high clustering coefficient.

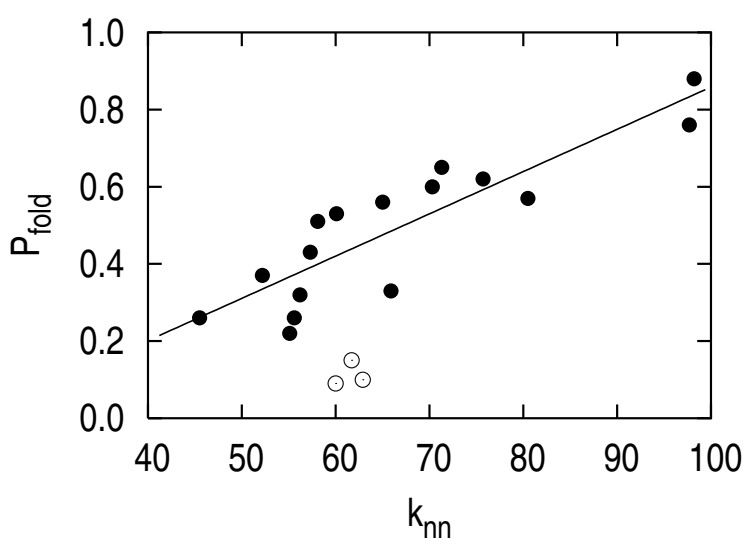

FIG. 5: Correlation between $P_{\text {fold }}$ and average neighbor connectivity $k_{n n}$. Three nodes used as a control (low connectivity/weight ratio and/or high clustering coefficient but similar fraction of native contacts) are shown with empty circles.

\section{CONCLUSIONS}

Complex network theory was used to analyze the conformation space of a structured peptide and the one of a random heteropolymer of same residue composition. Four main results have emerged. First, as it was already observed for a variety of networks as diverse as the WorldWide Web and the protein interactions in a cell, the con- 
formation space network of polypeptide chains is a scalefree network (power-law behavior of the degree distribution). Second, the native basin of the structured peptide shows a hierarchical organization of conformations. This organization is not observed for the random heteropolymer which lacks a native state. Third, free energy minima and their connectivity emerge from the network analysis without requiring projections into arbitrarily chosen reaction coordinates. As a consequence it is found that the denaturated state ensemble is very heterogeneous and includes high entropy, high enthalpy conformations as well as low entropy, low enthalpy traps. Fourth, the network properties were used to identify transition state conformations and two main average folding pathways. It was found that the average neighbor connectivity $k_{n n}$ correlates with $P_{\text {fold }}$, the probability of folding. $P_{\text {fold }}$ is computationally very expensive to evaluate. Hence, it will be important to generalize this result by analyzing other structured peptides which is work in progress in our research group. In conclusion, the network analysis seems particularly useful to study the conformation space and folding of structured peptides including the otherwise elusive transition state ensemble.

Acknowledgments We thank M. Cecchini, Prof. P. De Los Rios, E. Guarnera, Dr. E. Paci, Dr. M. Seeber and Dr. G. Settanni for interesting discussions. This work was supported by the Swiss National Science Foundation and the National Competence Center for Research (NCCR) in Structural Biology.
* corresponding author, tel: +41 163555 21, fax: +41 1 63568 62, e-mail: caflisch@bioc.unizh.ch

1 V. Daggett, A. R. Fersht, Is there a unifying mechanism for protein folding?, Trends in Biochemical Sciences 28 (2003) $18-25$.

2 J. Bryngelson, P. Wolynes, Intermediates and barrier crossing in a random energy-model (with applications to protein folding), J. Phys. Chem. 93 (1989) 6902-6915.

3 P. E. Leopold, M. Montal, J. N. Onuchic, Protein folding funnels: A kinetic approach to the sequence-structure relationship, Proc. Natl. Acad. Sci. USA. 89 (1992) 8721-8725.

4 M. Karplus, The Levinthal paradox: yesterday and today, Folding \& Design 2 (1997) S69-S75.

${ }^{5}$ H. Jeong, B. Tombor, R. Albert, Z. N. Oltvai, A. L. Barabási, The large-scale organization of metabolic networks, Nature 407 (2000) 651-654.

${ }^{6}$ R. Albert, H. Jeong, A.-L. Barabási, Diameter of the World-Wide Web, Nature 401 (1999) 130-131.

7 L.H. Greene, V.A. Higman, Uncovering network systems within protein structures, J. Mol. Biol. 334 (2003) 781791.

8 M. Newman, The structure and function of complex networks, SIAM REV. 45 (2003) 167-256.

9 J. Doye, Network topology of a potential energy landscape: A static scale-free network, Phys. Rev. Lett 88 (2002) 238701.

10 E. De Alba, J. Santoro, M. Rico, M. A. Jiménez, De novo design of a monomeric three-stranded antiparallel $\beta$-sheet, Protein Science 8 (1999) 854-865.

11 P. Ferrara, J. Apostolakis, A. Caflisch, Evaluation of a fast implicit solvent model for molecular dynamics simulations, Proteins: Structure, Function and Genetics 46 (2002) 2433.

12 P. Ferrara, A. Caflisch, Folding simulations of a threestranded antiparallel $\beta$-sheet peptide, Proc. Natl. Acad. Sci. USA. 97 (2000) 10780-10785.

13 A. Cavalli, P. Ferrara, A. Caflisch, Weak temperature dependence of the free energy surface and folding pathways of structured peptides, Proteins: Structure, Function and Genetics 47 (2002) 305-314.

14 A. Cavalli, U. Haberthür, E. Paci, A. Caflisch, Fast protein folding on downhill energy landscape, Protein Science 12
(2003) 1801-1803.

15 E. Ravasz, A.-L. Barabási, Hierarchical organization in complex networks, Phys. Rev. E 67 (2003) 026112.

16 R. Koradi, M. Billeter, K. Wüthrich, MOLMOL: a program for display and analysis of macromolecular structures, J. Mol. Graphics Modell. 14 (1996) 51-55.

17 B. R. Brooks, R. E. Bruccoleri, B. D. Olafson, D. J. States, S. Swaminathan, M. Karplus, CHARMM: A program for macromolecular energy, minimization, and dynamics calculations, J. Comput. Chem. 4 (1983) 187-217.

18 J. Gsponer, U. Habertür, A. Caflisch, The role of side-chain interactions in the early steps of aggregation: Molecular dynamics simulations of an amyloid-forming peptide from the yeast prion sup35, Proc. Natl. Acad. Sci. USA. 100 (2003) 5154-5159.

19 A. Hiltpold, P. Ferrara, J. Gsponer, A. Caflisch, Free energy surface of the helical peptide $\mathrm{Y}(\mathrm{MEARA})_{6}$, J. Phys. Chem. B 104 (2000) 10080-10086.

20 J. Gsponer, A. Caflisch, Role of native topology investigated by multiple unfolding simulations of four SH3 domains, J. Mol. Biol. 309 (2001) 285-298.

21 J. Gsponer, A. Caflisch, Molecular dynamics simulations of protein folding from the transition state, Proc. Natl. Acad. Sci. USA. 99 (2002) 6719-6724.

22 P. Ferrara, J. Apostolakis, A. Caflisch, Thermodynamics and kinetics of folding of two model peptides investigated by molecular dynamics simulations, J. Phys. Chem. B 104 (2000) 5000-5010.

23 W. A. Eaton, V. Munoz, J. Hagen, S, G. S. Jas, L. J. Lapidus, E. R. Henry, J. Hofrichter, Fast kinetics and mechanisms in protein folding., Ann. Rev. Biophys. Biomolec. Struc. 29 (2000) 327-359.

24 C. A. F. Andersen, A. G. Palmer, S. Brunak, B. Rost, Continuum secondary structure captures protein flexibility, Structure 10 (2002) 174-184.

25 R. Du, V. Pande, A. Grosberg, T. Tanaka, E. Shakhnovich, On the transition coordinate for protein folding, J. Chem. Phys. 108 (1998) 334-350.

26 J. Onuchic, N. Socci, Z. Luthey-Schulten, P. Wolynes, Protein folding funnels: The nature of the transition state ensemble, Folding \& Design 1 (1996) 441-450.

27 J. Schonbrun, K. A. Dill, Fast protein folding kinetics, 
Proc. Natl. Acad. Sci. USA. 100 (2003) 12678-12682.

28 S. R. Griffiths-Jones, M. S. Searle, Structure, folding, and energetics of cooperative interactions between the $\beta$ strands of a de novo designed three-stranded antiparallel $\beta$-sheet peptide, J. Am. Chem. Soc. 122 (2000) 8350-8356.

29 C. F. Wright, K. Lindorff-Larsen, L. G. Randles, J. Clarke, Parallel protein-unfolding pathways revealed and mapped, Nature Struct. Biol. 10 (2003) 658-662.

30 M. Vendruscolo, N. V. Dokholyan, E. Paci, M. Karplus, Small-world view of the amino acids that play a key role in protein folding, Phys. Rev. E 65 (2002) 061910-1-0619104, .

31 S. J. Demarest, Y. X. Hua, D. P. Raleigh, Local interactions drive the formation of nonnative structure in the denatured state of human alpha-lactalbumin: A high resolution structural characterization of a peptide model in aqueous solution, Biochemistry 38 (1999) 7380-7387.

32 A.-L. Barabási, R. Albert, Emergence of scaling in random networks, Science 286 (1999) 509-512.

33 M. Newman, The structure of scientific collaboration networks, Proc. Natl. Acad. Sci. USA. 98 (2001) 404-409.

34 H. S. Chan, K. A. Dill, Protein folding in the landscape perspective: Chevron plots and non-Arrhenius kinetics, Proteins: Structure, Function and Genetics 30 (1998) 233.

35 M. Karplus, Aspects of protein reaction dynamics: deviations from simple behaivor, J. Phys. Chem. B 104 (2000) $11-27$.

36 T. Lazaridis, M. Karplus, "New view" of protein folding reconciled with the old through multiple unfolding simulations, Science 278 (1997) 1928-1931. 
Supplementary Material
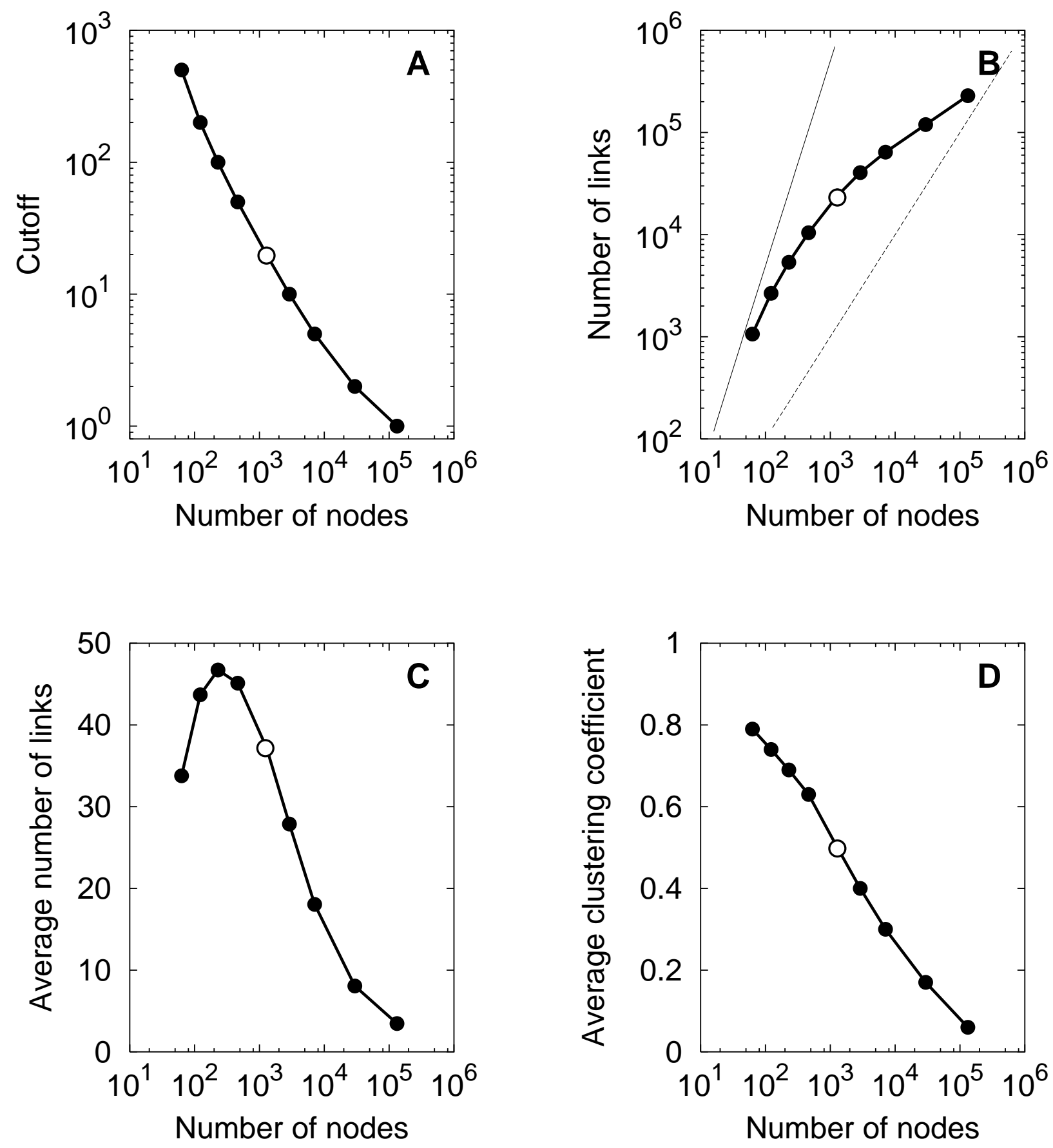

FIG. S1: Dependence of the beta3s network properties on the node-weight threshold. The threshold value used in the present work $(\tilde{w}=20)$ is shown as an empty circle while filled circles correspond to threshold values of, from left to right, 500, 200, 100, 50, 10, 5, 2 and 1. (A) Relation between the threshold value and the number of nodes. (B) Number of links as a function of the number of nodes. When the threshold is very large (i.e., small number of nodes) the network approaches a topology where all possible connections are present (solid line, $\left.N_{\text {links }}=N_{\text {nodes }}\left(N_{\text {nodes }}-1\right) / 2\right)$. When the threshold is small (i.e., large number of nodes) the network approaches a topology with only one link per node (dashed line, $N_{\text {links }}=N_{\text {nodes }}$ ). (C) Average number of links per node $\langle k\rangle$ as a function of the number of nodes. (D) Average clustering coefficient $C$ as a function of the number of nodes. 


\section{Supplementary Material}
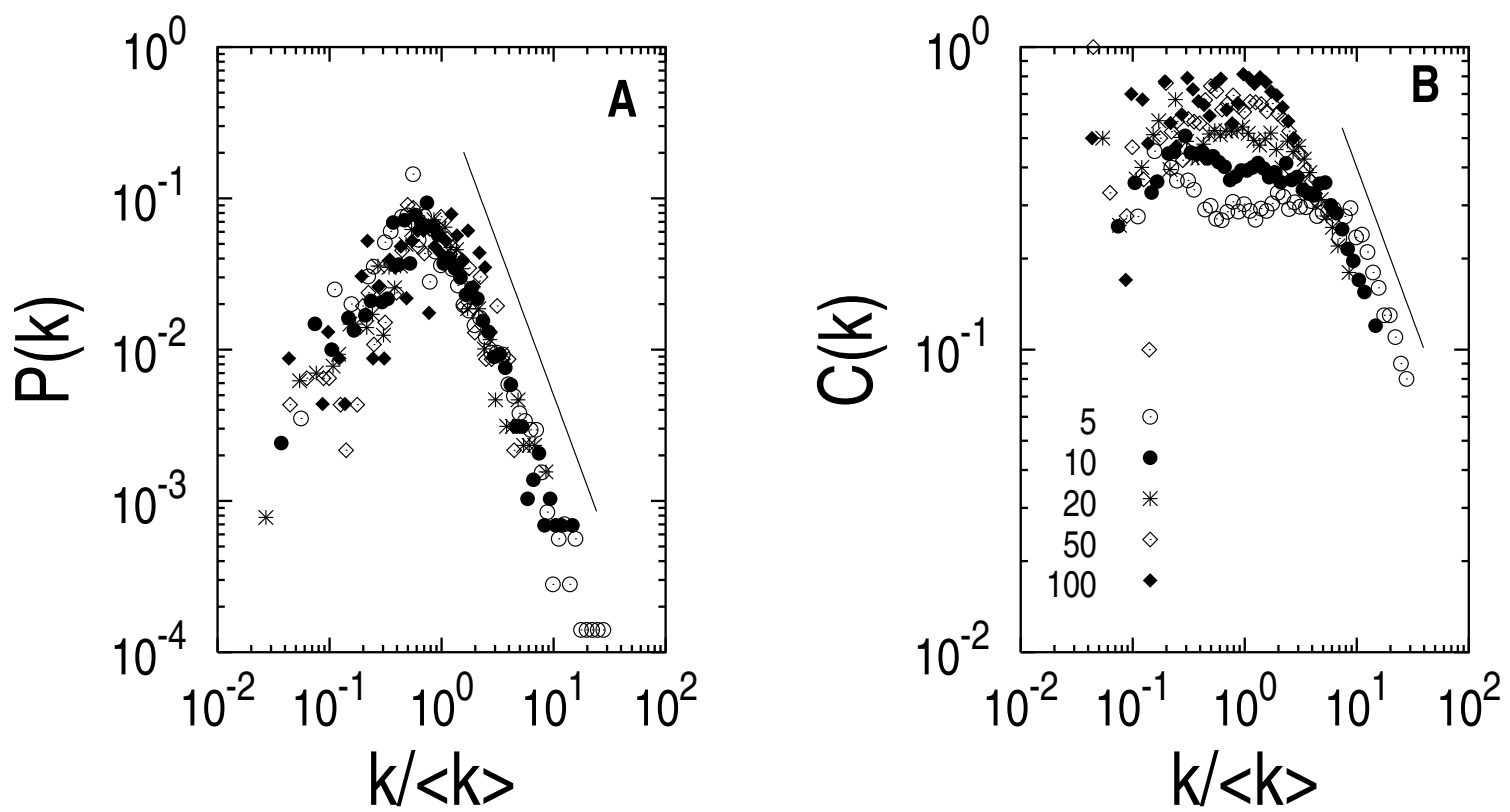

FIG. S2: Dependence of the beta3s connectivity distribution (A) and clustering coefficient distribution (B) on the node-weight threshold. This plot shows that the scale-free behavior and the $1 / k$ tail of the clustering coefficient distribution are robust with respect to the choice of threshold values.
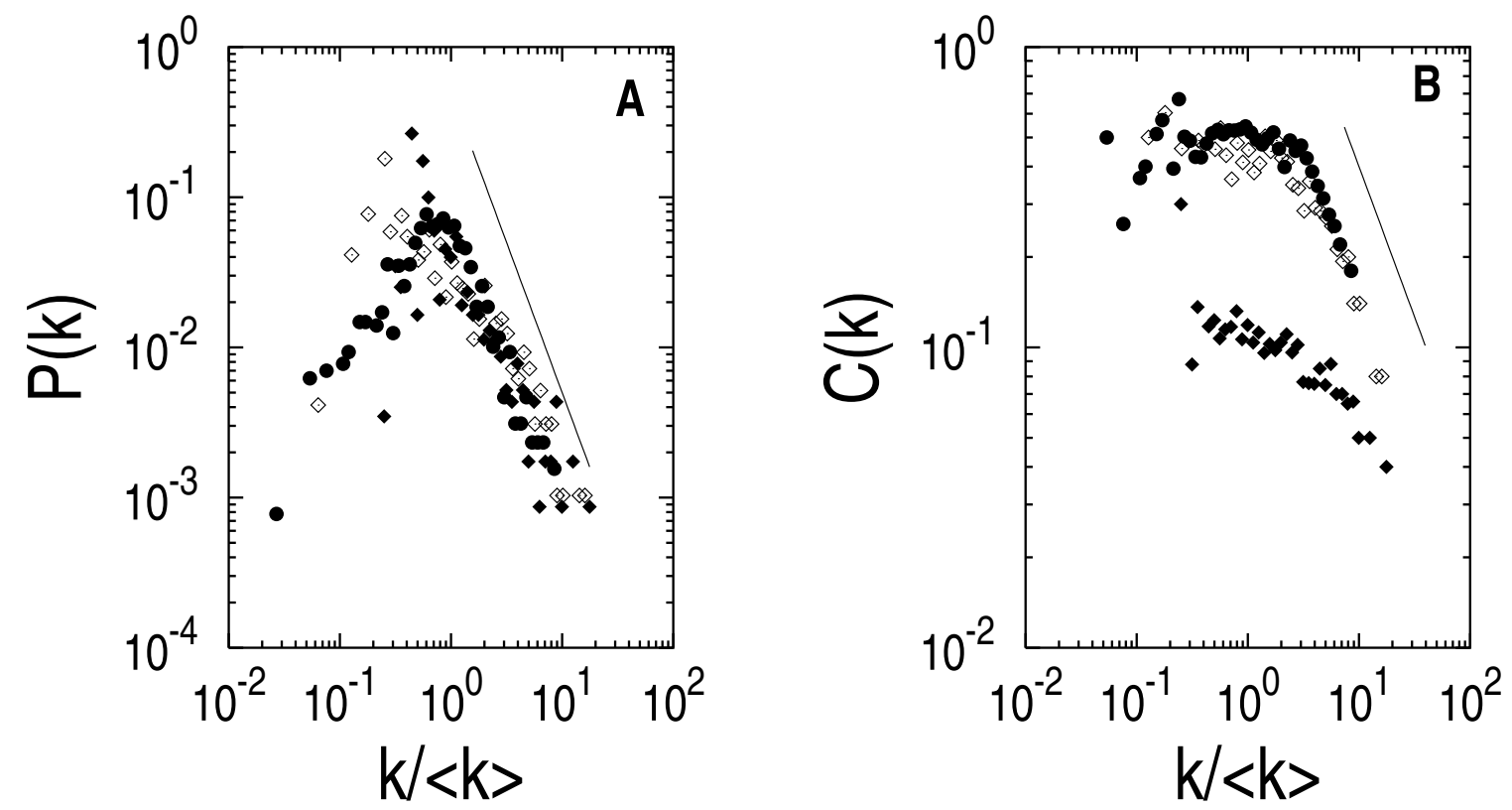

FIG. S3: Connectivity distribution (left) and clustering coefficient distribution (right) for beta3s (filled circles), another structured peptide, i.e., residues 101-111 of $\alpha$-lactalbumin (empty diamonds, Demarest et al., (1999) Biochemistry, 38, 7380), and a 20-residue homo-glycine which is unstructured (filled diamonds). 


\section{Supplementary MATERIAL}

TABLE S1: Supplementary material. Nodes used for $P_{\text {fold }}$ evaluation.

\begin{tabular}{|c|c|c|c|c|c|c|c|c|c|c|}
\hline $\begin{array}{c}\text { Node } \\
\text { number }\end{array}$ & $\begin{array}{l}\text { Probabili } \\
\text { of } \\
\text { folding } \\
P_{\text {fold }}\end{array}$ & $\begin{array}{l}\text { ystandard } \\
\text { devia- } \\
\text { tion } \\
\sigma_{P_{\text {fold }}}\end{array}$ & $\begin{array}{c}\text { Neighbor } \\
\text { connectivity } \\
k_{n n}\end{array}$ & $\begin{array}{c}\text { Weight } \\
\tilde{w}\end{array}$ & $\begin{array}{c}\text { Number } \\
\text { of links } \\
k\end{array}$ & $k / 2 \tilde{w}$ & $\begin{array}{l}\text { Clustering } \\
\text { coeffi- } \\
\text { cient } \\
C\end{array}$ & $\begin{array}{l}\text { Native } \\
\text { con- } \\
\text { tacts } \\
Q\end{array}$ & $\begin{array}{c}\text { Standard } \\
\text { devia- } \\
\text { tion } \\
\sigma_{Q}\end{array}$ & $\begin{array}{l}\text { Secondary } \\
\text { structure } \\
\text { string }\end{array}$ \\
\hline 432 & 0.22 & 0.17 & 55.1 & 54 & 40 & 0.37 & 0.31 & 0.38 & 0.09 & -----SS-EEEEESSEEEE- \\
\hline 218 & 0.26 & 0.20 & 45.5 & 105 & 73 & 0.35 & 0.23 & 0.42 & 0.09 & ----SSS---EEESSEEE-- \\
\hline 313 & 0.26 & 0.16 & 55.6 & 70 & 60 & 0.43 & 0.28 & 0.46 & 0.09 & -EEE-STTEEE-SSS----- \\
\hline 446 & 0.32 & 0.25 & 56.2 & 52 & 50 & 0.48 & 0.28 & 0.40 & 0.10 & --EEESSEEE---SS----- \\
\hline 308 & 0.33 & 0.28 & 65.9 & 72 & 69 & 0.48 & 0.23 & 0.44 & 0.08 & ----SSS--EEEESSEEEE- \\
\hline 315 & 0.37 & 0.26 & 52.2 & 70 & 60 & 0.43 & 0.24 & 0.47 & 0.08 & -EEE-STTEEE--SS----- \\
\hline 306 & 0.43 & 0.27 & 57.3 & 73 & 60 & 0.41 & 0.31 & 0.42 & 0.08 & -----SS--EEE-STTEEE- \\
\hline 208 & 0.51 & 0.26 & 58.1 & 115 & 87 & 0.38 & 0.23 & 0.43 & 0.08 & -----SS--EEEESSEEEE- \\
\hline 589 & 0.53 & 0.31 & 60.1 & 40 & 52 & 0.65 & 0.17 & 0.45 & 0.10 & ---SSSTT-EEEESSEEEE- \\
\hline 580 & 0.56 & 0.34 & 65.0 & 40 & 47 & 0.59 & 0.26 & 0.48 & 0.08 & -EEEESSEEEE--SSS---- \\
\hline 197 & 0.57 & 0.39 & 80.5 & 121 & 105 & 0.43 & 0.28 & 0.52 & 0.09 & -----STT-EEEESSEEEE- \\
\hline 540 & 0.60 & 0.16 & 70.3 & 44 & 49 & 0.56 & 0.28 & 0.46 & 0.07 & -----GGG-EEE-STTEEE- \\
\hline 285 & 0.62 & 0.30 & 75.7 & 80 & 68 & 0.42 & 0.30 & 0.47 & 0.07 & -----GGG-EEEESSEEEE- \\
\hline 630 & 0.65 & 0.22 & 71.3 & 38 & 56 & 0.74 & 0.29 & 0.44 & 0.11 & -----STT--EE-STTEE-- \\
\hline 426 & 0.76 & 0.20 & 97.7 & 55 & 76 & 0.69 & 0.43 & 0.55 & 0.12 & ---B-TTTB-EEESSEEE-- \\
\hline 280 & 0.88 & 0.18 & 98.2 & 82 & 81 & 0.49 & 0.43 & 0.56 & 0.09 & ---EESSEE-EE-STTEE-- \\
\hline \multicolumn{11}{|c|}{ Control simulations } \\
\hline 174 & 0.09 & 0.10 & 60.0 & 139 & 51 & 0.18 & 0.61 & 0.37 & 0.07 & --EE-STTEEEESTTEEEE- \\
\hline 179 & 0.09 & 0.10 & 25.0 & 135 & 19 & 0.07 & 0.33 & 0.44 & 0.07 & --EESSEE--EEESSEEE-- \\
\hline 15 & 0.10 & 0.13 & 35.8 & 3243 & 73 & 0.01 & 0.28 & 0.47 & 0.08 & --EEESSSEEEEESSEEEE- \\
\hline 200 & 0.10 & 0.27 & 62.9 & 119 & 51 & 0.21 & 0.31 & 0.31 & 0.07 & ----BSSB--EEESSEEEE- \\
\hline 475 & 0.15 & 0.17 & 61.7 & 48 & 34 & 0.35 & 0.68 & 0.43 & 0.06 & -EEE-STTEEETTTT-EEE- \\
\hline
\end{tabular}

